believe these cases are so acute that they offer a more hopeful field for the use of serums than vaccines. With serium treatment many good results have been recorded. Ulcers of the cornea may be due to other organismsBacillus coli, diplobacillus, etc.-but I can find no records of such cases treated by means of vaccines.

As an accessory to vaccine treatment, the local light treatment by cadmium-zinc lamp, described by Hertel, ${ }^{9}$ is valuable. He claims that the treatment causes (i) direct bactericidal action; (ii) indirect effects-con- abscess, or retinal phlebitis. Such cases may be secondary to skin abscesses, puerperal infections, pleuropneumonia etc., and the causative organisms are usually staphylococci or streptococci. Another variety of metastatic infection is irido-cyclitis secondary to pyorrhoea alveolaris (Adams ${ }^{12}$ ), where staphylococcus or preumococcus is the infecting agent. In all such cases vaccines can be used with good hope of success, cultures being obtained from the primary focus of disease.

On the other hand, in internal infections of the eye without obvious lesions elsewhere the diffi culty arises of obtaining enltures of the causative organism and making a becteriological diagnosis.

However, in cases of irido-cyclitis cultures have been made from the aqueous after paracentesis by Mayou, ${ }^{3}$ and he reports a case in which Staphylococcus albus was grown, and the irido-cyclitis cured by vaccine, using doses of 1,000 million. Twe other similar cases of irido-cyclitis treatcd by staphylococcus vaccine are recorded by the same author, one being cured, the othes relieved. Weeks ${ }^{13}$ records two cases of gonococcal iritis treated by stock vaccine with success, and he mentions two similar cases treated by C. A. Oliver, and one, recorded by Posey, of severe gonococcai uveitis which improved. Streptococcus has been cultivated by other workers from the anterior chamber in cases of irido-cyclitis.

The general rule must be laid down that larger doses of vaccine are nøcessary ' in internal diseases of the eye owing to the peculiarities of its blood supply.

Chart 7,-Case E. P. A, 10 million of "stock" streptococcus vaccine. B, 5 -million of specific vaccine; c, 15 million ditto

junctival and ciliary injection, oedema, and cellular infiltration of the cornea.

Here, then, we have a means for producing (1) increased passage of serum to the diseased part, (2) increased local leucocytosis, (3) antoinoculation. If we raise the opsonic index by vaccines, such treatment should give even better results than in the hands of Hertel using the light alone (25 cures out of 47 cases of serpent ulcer of the cornea)

Post-operative Infection.

In post-operative infection excellent results have been obtained with vaccines. Cultures must be taken from the wound, and a vaccine used as early as possible. Staphylococci, streptococci, and pneumococci are the commonest infecting organisms.

Staphylococcus.-Maddox ${ }^{10}$ reports an infected case of cataract extraction, with pus along line of incision; vitreous grey and turbid, and iris "rotten-looking." In jection of staphylococcus vaccine gave rapid improvenent, followed by cure.

Streptococcus. - I have obtained a very striking result by vaccine in a case of late post-operative infection by streptococcus.

E. P., July 13th, 1908 : Lids healthy. Right preliminary iridectomy performed. July 19th: Much ciliary injection. Th inflammation became rapidly worse, with intense chemosis, the elges of the incision oedematous, iris discoloured, and a hrpopron developed. - Cultures from wound showed pure growth of streptococcus. July 22 nd : Increased ciliary injection gnd bopon. July 23rd: Less chemosis. July 24 th : Hypopyon disappeared, injection and chemosis less. July 29th : Aqueous clear.

August 8th. A small hypopyon reappeared, lasting until August 14th. The inflammation gradually subsided, and the were performed later, without complication, and vision $\{$ was were perfor

obtained.
I give the details of treatment and opsonic indices, in the above chart.

We see, then, that vaccine treatment is of great value in post-operative infections, the prognosis of which has been iio bad in the past.

Internal Infections.

In internal disease of the eye due to pyogenic organisms the obtaining of a bacteriologicál diagnosis is difficult: The disease may be primary or secondary to a focus elsewhere in the body. Lesions of the eye secondary to disease of other parts have been described by Holmes Spicer, ${ }^{11}$ and may take the form of irido-cyclitis, retinal
Lastly, mention may be made of a result obtained by Trantes ${ }^{15}$ who used cow-pox vaccine in a case of trachoma with resulting cure; the experiment was suggested by the observation of a case of trachoma which became well after an attack of small-pox.

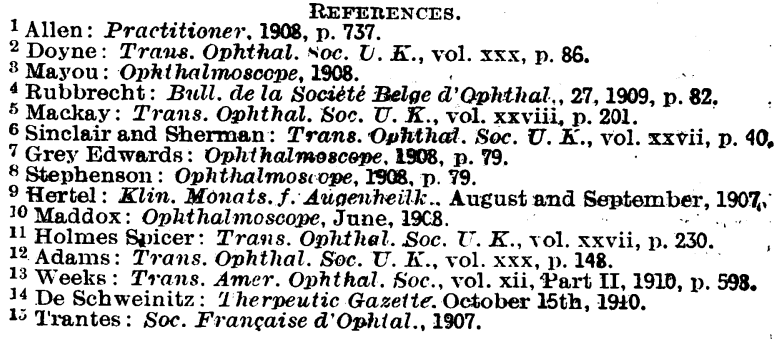

\section{A CASE OF ADDISON'S DISEASE TREATED WITH TUBERCULIN.*}

BX. JOHN M. H. MUNRO, D.Sc., M.R.C.S., L.R.C.P.,

ASSISTANT FOR OPSONIN TREATMENT IN THE PATHOLOGICAT DEPARTMENT, BRISTOL IBOYAL INFTRMARY.

A weLl-EDUCATED and intelligent lady, aged 34, un. married, consulted me in April, 1907, under the following circumstances.

Two years earlier, when in good health and having had no serious illness, she began to ail and has got gradually worse. She complained of lassitude and want of strength, whereas she was formerly fond of getting about and of occupation; said she had no appetite, but forced herself to eat. She had frequent headaches and suffered from constipation, varied during the last six months by a few attaeks of diarrhoea; also had backaches (lumbar), which have been more frequent since last winter. She suffers from post-nasal eatarrh, and the throat is sometimes sore, but she has no congh. Used to sing much, but has gradually discontinued it during the last two years; formerly could cycle 20 miles, but gave this up last summer. The hands and legs had been

* A paper read at Bath, April 28th, 1909, at the meeting of the Bath and Bristol Branch of the British Medical Association. Postscript 
shaky for about two years, and the temper was getting irritable. She complained much of nausea, without vomiting, dating from six months back. It came on at any time and was not related to food. Sometimes when walking there was a feeling of unreality, and a sensation as if she would stagger at any moment. She fainted a few mornings since for the first time. Latterly there had been much mental depression. A month ago she first noticed a mottled brown appearance of the skin on the thighs, and later herself and friends had remarked it round the mouth and in other spots. There had been a dull pain, not always present, between the clavicle and the right breast, since last winter only. For many years the right knee had been painful after exercise.

It will be noticed that some of these symptoms were quite recent, and all except the last appeared since two years before. At that time a younger sister, to whom she was much attached, died at Mentone of phthisis after ten years' illness. The patient nursed her a good deal during the earlier part of the time, and went out to Mentone to be with her just before the end. Felt the shock very much, and dates her own illness from that event. Eighteen months ago she consulted a doctor, who said that her organs were quite' sound, that she had nervous breakdown, and advised her going abroad. This was not practicable, and she got worse instead of better; has tried iron and other tonics without effect.

With the exception of the sister who died there is no immediate family history of tuberculosis. Her father is English, her mother a native of Guernsey; two brothers and one sister are alive, all in fair health.

\section{Condition when First Seen.}

On examination the patient is a tall, well nourished, somewhat sallow brunette, of naturally alert temperament. The pulse varies from 76 to 84 , is feeble, and occasionally a little irregular. The tongue is furred at the back; there is little or no tremor. She is of abstemious habit.

The throat, although patchy and not quite healthy in appearance, shows no marked abnormality. No physical signs of disease were elicited by the usual examination of the thoracic and abdominal viscera, and examination per vaginam, digital and specular, also gave negative results. Menstruation begal at 14, was regular until a year back, lasting five days, but since then the intervals are fourteen days only. The urine is normal in quantity and colour, acid, specific gravity 1020, no albumen or glucose, slight cloud of mucus, no bacterial turbidity. Ocular, plantar, and tendon reflexes are normal.

There is a decided fine tremor of hands and legs, but no There is a decided fine tremor of hands and legs, but no
tenderness. No enlargement of thyroid, or thrill, or extenderness. No enlargement of thyroid, or thrill, or exjunction of the second rib and cartilage on the right side, very junction of the second rib and cartilage on the right side, very slightly tender; this is the seat of the pain referred to, and is
believed by the patient to be recent. The body is well nourished believed by the patient to be recent. The body is well nourished,
weight 9 st. 2 l lb., muscles remarkably firm in contrast with weight 9 st. 2 l lb., muscles remarkably firm in contrast with subsequently measured by consultant as one-third normal. The right leg is $1 \frac{1}{2}$ in. shorter than the left, measuring from the anterior superior :iliac spine to the external malleolus; the shortening is between hip and knee, and on inspection the pelvis is tilted to the right; movements of the knee and hip joints are perfect, but the ache experienced in the richt knee after exercise was felt also after the examination.

An abnormal body odour which used not to exist is now, at times, perceptible to others. The recent brown pigmentation of the skin is most noticeable in irregular patches on the chin, lower and upper lips, sides of nose and forehead (very recent there), upper and inner parts of thighs, on buttocks round anus, on neck, forearms, and shoulder (ring round exposed portion), it is traceable on mucous surfaces of mouth, conjunctivae, and genitals, and on palmar as well as dorsal aspect of hands. It appears to be diffuse or general, but deeper in places, and is appears to be diffuse or general, but deeper in places, and is
noticeably interrupted on thighs, backs of knees, and some other parts by oval and irregular patches of leucoderma. Although the patient is a brunette, she is confident that until recently the normal tint of her skin was nearer that of the light patches than the rest.

A blood examination showed over 100 per cent. haemoglobin, erythrocytes normal in number and shape, polynuclear leucocytes 4,200 , no abnormal cells. The opsonic index to human tubercle, carefully determined in my own laboratory, was 0.80 , two days later 1.00, but heated serum index (thermo-stabile opsonin) was 0.40 . The slight variation from normal in the that opsonin index can hardly be called significant, but the thermo-stabile opsonin index is. A heated serum index of 0.0 is quite consistent with tuberculosis, but I have never found a high one -anything like as high as 0.40 -in a case that was not undoubtedly tuberculous or highly suspicious. The post-nasal mucus was free from tubercle bacilli; and this I have found have treated with tuberculin with marked benefit.

Although I embarked on this case with a strong disposition to look for latent or early phthisis, the clinical picturè and history as above disclosed did not seem as a whole explicable by any diagnosis but that of Addison's disease, of tuberculous origin, with the most objective symptom-pigmentation-quite recent, and with possibly tuberculous foci outside the suprarenals also. We have the initial mental shock, the tuberculous contact, the slow progressive character of the early stage, the myasthenia without muscular atrophy, nausea without ascertainable cause, occasional diarrhoea, fine tremor, signs of feeble circulation, progressive debility, languor and depression, the absence of any other organic disease to account for this group of symptoms, and, finally, the later onset of the pigmentation to clinch the whole. If we look upon this last as simply patches of lencoderma in a naturally dark skin, with heaped-up pigment ncar the margins of the cleared areas to give the darker effects, we are left without explanation of the accompanying group of symptoms. The patient's own evidence and the occurrence of mucous and palmar pigmentation stamp the deposit as pathological; and, moreover, the liairs on the lighter areas are normally pigmented and not bleached, as in ordinary cases of leucoderma. If we suppose tuberculosis with pigmentation but with suprarenals unaffected, we are at a loss to locate the lesions beyond the node of the second rib and the very doubtiul knee or hip symptom dating from childhood, and again find in them nothing to account for the very grave condition of illness undoubtedly present. Other known causes of pigmentation are either absolutely excluded or exceedingly unlikely. On the known fact that the great majority of cases of Addison's disease which have been examined post mortem show tuberculous lesions in the suprarenals and on the apparently early stage of this case, $I$ proposed to try tuberculin treatment supplemented by surrarcual extract. On April 12th. I took the patient to Dr. H. D. Rolleston for an opinion, and he could suggest no alternative diagnosis and endorsed the treatment with suprarenal extract. $\mathrm{He}$ drew my attention to notes of many cases of tuberculosis with pigmentation, and also to the recognition by French physicians of a condition clescribed as "Addisonism"- that is, a clinical picture agreeing with Addison's disease, but with symptoms mild in degree, and lacking the post-mortem confirmation of suprarenal lesion, therefore presumably differing only in degree, and possibly offering a less unfavourable prognosis. We cheered the patient with this and with the fact that the arterial pressure as taken by Dr. Rolleston was $120 \mathrm{~mm}$. mercury. As regards tuberculin, he agreed that I might try it, and I saw Sir A. Wright the same afternoon; he strongly recommended me to try tuberculin, and also pointed out two or three cases of obvious tuberculous lesions with pigmentation amongst his out-patient records.

As regards blood pressure, my Riva-Rocci gauge was a.t the instrument-makers at this time, but soon after and many times since. I found the pressure to vary littlc, 110 to $115 \mathrm{~mm}$. on the first four occasions and much the same since; 103 was recorded on two occasions each after a few weeks' absence from treatment; 118 also twice, first in June, 1908, and again in September, about which time the most marked improvement set in. Since certainly in some fatal cases of suprarenal disease the blood pressure does not fall markedly until-within a short time of death, the absence of an abnormally low pressure in this case does not tell against the diagnosis.

The patient was left in Bath under my care, and has been under treatment for two years (April, 1909) with three intervals of a few weeks spent at her home. On April 13th, 1907, the first injection of ${ }_{13}^{1} 60 \mathrm{mg}$. T.R. human was given, the opsonic index being 1.05. Next day patient felt. worse, the pain in the rib had increased, and there was a little diarrhoea. Thirty-two hours after injection the opsonic index rose to 1.48. Such a response, provided it is based on careful uniform determinations in one's own laboratory, has been in my experience, which on this point is entirely confirmatory of Sir A. Wright's, of great value in confirming the existence of a bacterial infection, and, in hopeful cases, of foreshadowing improvement. Several scores of indices have been subsequently taken in this case for the of indices have been subsequently taken in this case for the
sake of research and in the hope of controlling the inoculations; sake of research and in the hope of controlling the inoculations,
but they may be disposed of by saying that the index was very but they may be disposed of by saying that the index was very
rarely above normal or below 0.8 , and that after a short time it rarely above normal or below 0.8 , and that after a short time it
was noticed that the patient's serum acquired the power of
agglutinating the erythrocytes of the emulsion used (generally agglutinating the erythrocytes of the emulsion used (generally
mine and iny assistants's). This phenomenon, which cannot fail to strike the eye of the operator by the curdled appearance of the mixture of corpuscles, bacterial emulsion and serum in the pipette, sometimes before, sometimes after the fifteen 
the index determinations (see Fleming's paper in Practitioner' May, 1908). My patient's serum would retain this property for months in succession and then lose it.

The pain in the rib ceased on the second day after the first inoculation, and returned only at rare intervals and in a very transient manner. On the ninth day the nausea was reported transient manner. On the ninth day the nausea was reported as almost nil, occurring only once or twice in a slight degree. lay, but on the ninth the notes read, "On the whole, decidedly better." The effects, if any, of a first injection of bacterial better." The effects, if any, of a first injection of bacterial vaccine being very important to observe, administration of suprarenal extract was deferred until the above notes were recorded. Fifteen grains daily were then given for a period of three months, and as the tuberculin injections were at first at intervals of ten days or more, it was fairly easy to form a judgement as to their relative effects. At first the suprarenal extract was thought to have some influence in preventing the return of nausea, but at the end of three months, no noticeable result appearing attributable to this remedy, it was discontinued.

The dose of tuberculin was after a time increased to 1 mg The dos of tuberculin was after a time increased to and this was eventually given weekly for several months. During the first year, at any rate, almost every injection produced some malaise for twenty-four hours; afterwards this were tolerated well with good results, whilst for the last two months doses up to $\frac{1}{50} \mathrm{mg}$. have beev given every fourth and lately every second day: Slow but sure progress was apparent from the first, and this was sufficient to ensure the patient's return to Bath after two or three home visits of a month each, luring which the improvement was scarcely maintained. The asthenic symptoms, and rarely nausea, were still complained of, but about six months ago much more rapid improvement set in, the patient began to say she felt more like he: old self, and contemporaneously with this it became apparent that the patches of leucoderma were getting larger and running together, and that fresh areas of clearance were presenting. A photograph was secured last September, and another was taken last week. On comparing them this point will be easily recognized.

The present condition (April, 1909) is that the pain in rib, after becoming less and less frequent, has not been felt for after becoming less and less frequent, has not been felt for three months; the knee still aches after walking, but long there are no attacks of nausea or diarrhoea; the mental there aro no attacks of nausea or diarrhoea; the mental lepression has within the last six months entirely disappeared; the tremor and stagge:ing disappeared eighteen months ago gone; the catamenial intervals have become normal; and the post-nasal discharge, which used to fill half an egg-cup each morning, is less than half this quantity.

Turning from clinical to economical considerations, the improvement will be more readily credited. The mother having lost one daughter from phthisis after all sorts of treatment, wished this one to remain at home and take her chance uncler good climatic conditions when she learnt the usual disappointing results of our ordinary therepentic measures in Addison's disease. At each home visit of the daughter the mother became more willing that the tuberculin treatment should be continued, and now writes me that the patient is "quite her own old noisy self again," and consents to a still further extension of the treatment if I think it necessary. The patient, however, feels quite well enough to resume her old life and home duties, and it is so arranged. She objects to take tuberculin by the mouth or hypodermically without my supervision, and will resume the trial of suprarenal substance. As in a favourable event the case will pass out of my hands, it seems a fitting time to record it. As to prognosis; I am not able to say a word.

Postscript, May 18t, 1911.-At the discussion on the above paper no one questioned the diagnosis. In conre rsation Mr. Pagan Lowe remarked to me that, whilst thoroughly agreeing with the diagnosis, he would like to khow the further history of the case in, say, two years' time. Feeling myself that much of the interest lay in the future, I decided to suspend publication. I now publish the paper as read, and am able to add the following note, having just had an opportunity of examining the patient during a short visit to Bath :

The second trial of suprarenal extract was terminated, like the first, after a couple of months, as it was thought to disagree, and no definité favourable effect was noticed. By September 1909 , the patient was definitely not so well, and returned to Bath for four months, during which she had thirty-four inoculations of $1 . \mathrm{mg}$. T.R. She returned home very well in January, 1910 , and since then has paid only three short visits to BathMarch 28th to April 7th, 1910; November 14th to December 20th, 1910; and April 6th to 25 th, 1911 ; receiving six, twelve and four inoculations at the several visits. For the six, twelve month thato past fifteen month, therefore, she has remained in good health and spirits duties, and her general condition has decidedly ímproved instead of showing a tendency to fall back. Present condition (April 25th 1911): Pulse 72, now and then a little irregular; no bruit; blood pressure $108 \mathrm{~mm}$.; no nausea, no tremor; hand grips normal ; weight 9 st. $8 \mathrm{lb}$. ; Rarely has headaches, and gets about as of weight 9 st. $8 \mathrm{lb}$. Rarely has headaches, and gets about as of
old. Has had a trying winter with nursing, etc. There is not much change in the outlines of the lighter areas (that is, a much change in the outlines of the lighter areas (that is, a photograph would resemble the second of the two former ones), but there is a noticeable further general lightening of the pigmentation, so that friends tell her the face is much less dark
than even a year ago. Palmar pigmentation, and that of the than even a year ago. Palmar pigmentation, and that of the

Postscript, March 7th, 1912.-The patient remains quite well, and little further change has taken place in the details of the pigmentation, which is, however, generally lighter.

The cure has not suffered interruption in spite of the anxious home conditions occasioned by the illness and death of the patient's father.

[Photographs sent by the author show the pigment change, but are not sufficiently strong to give satisfactory reproductions.]

\section{STATISTICS OF A SERIES OF EIGHTY-SIX CASES OF PNEUMONIA, WITH A NOTE ON ALCOHOL IN THE TREATMEN'T.}

\section{By ARTHUR T. JONES, M.R.C.S., L.R.C.P.LOND.} MOUNTAIN ASH.

IT would doubtless be interesting if general practitioners were to record series of cases of the more common ailments occurring in their practices, for I am sure that there are many important factors that may be observed and notified as being useful and helpful from a point of view of diagnosis and treatment as it presents itself to the general practitioner, and to compare them with statistics as given in textbooks, which, as a rule, are statistics of cases seen and treated in hospital practice, and thus it becomes interesting to know whether cases treated in general practice and hospital practice give similar results, or whether they differ very materially, and whether one may deduce any points which would account for the different results obtained, if there are any. With this object in view I beg to record a series of cases of pneumonia which have occurred in my practice. The cases, which were of the lobar type, are 86 in number, and are tabulated as follows:

Eighty-six Cases-54 Males and 32 Females.

\begin{tabular}{|c|c|c|c|c|c|}
\hline Age. & & & $\begin{array}{l}\text { No. of } \\
\text { Cases. }\end{array}$ & $\begin{array}{c}\text { No. of } \\
\text { Deaths. }\end{array}$ & $\begin{array}{c}\text { Per- } \\
\text { centage. }\end{array}$ \\
\hline Under 10 years of age & $\cdots$ & ... & 10 & 3 & 30 \\
\hline Between 10 and 20 years & $\cdots$. & ... & 11 & 1 & 9 \\
\hline Between 20 and 40 years & ... & $\cdots$ & 39 & 9 & 23 \\
\hline Between 40 and 60 years & ... & $\cdots$ & 21 & 7 & 33 \\
\hline Over 60 years of age & $\cdots$ & $\cdots$ & 5 & 3 & 60 \\
\hline Total $\ldots$ & $\cdots$ & $\cdots$ & 86 & 23 & 26 \\
\hline
\end{tabular}

From these facts it will be seen at once that the disease is more common in males than females, and it occurs more frequently between the ages of 20 and 40 , and that the mortality-rate increases with each decade, with the excep tion that the rate of mortality under 10 years of age is high, but the highest being over 60 years of age, it being at that period over 60 per cent. The average mortalityrate in the series is 26 per cent., and this compares favourably with the statistics given by Professor Osler in his Textbook of Medicine. Professor Osler says: "Hospital statistics show that the mortality-rate ranges from 20 to 40 per cent. Of 1,012 cases at the Montreal-General Hospital the mortality was 20.4 per cent. Of 3,969 cases treated at the Charity Hospital, New Orleans, the deathrate was 38 per cent. Our mortality at Johns Hopkins Hospital was 25 per cent. in the whites and 30 per cent. in the coloured. In 704 cases at the Pennsylvania Hospital the mortality was 29 per cent., and at Boston City Hospital it was 29 per cent. in 1,443 cases. The mortality-rate in private practice. varies greatly... R. V. Howard treated 